\title{
Exact Pair Theorem for the $\omega$-Enumeration Degrees
}

\author{
Hristo Ganchev * \\ Sofia University, Faculty of Mathematics and Informatics \\ 5 James Bourchier blvd. 1164 Sofia, Bulgaria \\ h.ganchev@gmail.com
}

\begin{abstract}
In the paper the exact pair theorem for the $\omega$-enumeration degrees is proved. As a corollary an exact pair theorem involving the jump operation for the enumeration degrees is obtained.

Mathematics subject classification: 03D30

Key words and phrases: $\omega$-enumeration degrees; enumeration degrees; exact pair; jump.
\end{abstract}

\section{Introduction}

The $\omega$-enumeration degrees and the structure $\mathcal{D}_{\omega}$ are introduced by Soskov in [2]. In order to define it we first consider the set of all denumerable sequences of sets of natural numbers, which we will denote by $\mathcal{S}$. In $\mathcal{S}$ we define the reflexive and transitive relation " $\leq_{u}$ " and the equivalence relation " $\equiv_{u}$ " as follows: for any two sequences $\mathcal{A}, \mathcal{B} \in \mathcal{S}$

$$
\mathcal{A} \leq_{u} \mathcal{B} \Longleftrightarrow J_{\mathcal{B}} \subseteq J_{\mathcal{A}}, \quad \mathcal{A} \equiv_{u} \mathcal{B} \Longleftrightarrow J_{\mathcal{A}}=J_{\mathcal{B}}
$$

where $J_{\mathcal{A}}$ is the set of the Turing degrees of all $X \subseteq \mathbb{N}$ such that $(\forall k)\left(A_{k}\right.$ is r.e. in $X^{(k)}$ uniformly in $k$ ). Since we have that $A \equiv_{u} \mathcal{B} \Longleftrightarrow \mathcal{A} \leq_{u} \mathcal{B} \& \mathcal{B} \leq_{u} \mathcal{B}$, by factorizing the structure $\left(\mathcal{S}, \leq_{u}\right)$ with respect to $\equiv_{u}$, we obtain the structure $\left(\mathcal{D}_{\omega}, \leq_{\omega}\right)$, where the factor relation $\leq_{\omega}$ is a partial order. By $d_{\omega}(\mathcal{A})$ we shall denote the equivalence class generated by the sequence $\mathcal{A}$.

In [2] it is shown that $\mathcal{D}_{\omega}$ is an upper semi-lattice with least element, that the $\Sigma_{2}^{0} \omega$-enumeration degrees are dense and that there is no minimal $\omega$-enumeration degree. A notion of a jump operator in $\mathcal{D}_{\omega}$ is also introduced. It is shown that the substructure $\mathcal{D}_{1}=\left\{d_{\omega}(\mathcal{A}) \mid A_{n}=\emptyset\right.$ for $\left.n>0\right\}$ is isomorphic to the semi-lattice $\mathcal{D}_{e}$ of the enumeration degrees.

From the omitting theorem proved in [3] it follows that every $\omega$-enumeration degree is the greatest lower bound of two $\omega$-enumeration degrees strictly above it, i.e., every $\omega$-enumeration degree has a minimal pair.

In this paper we shall prove that every monotone increasing sequence of $\omega$-enumeration degrees has an exact pair.

\footnotetext{
^ This work was partially supported by Sofia University Science Fund.
} 
Definition 1 Let $(X, \leq)$ be a partially ordered set and let $a_{0} \leq a_{1} \leq \cdots \leq a_{n} \leq$ $\ldots$ be an increasing sequence in $X$. We say that $f, g \in X$ form an exact pair for $\left\{a_{n}\right\}_{n<\omega}$ if the following two conditions hold:

(i) $(\forall n)\left(a_{n} \leq f, g\right)$;

(ii) $x \leq f, g \Longrightarrow \exists n\left(x \leq a_{n}\right)$.

The existence of exact pairs for the Turing degrees and for the enumeration degrees is proved by Spector [4] and Case [1] respectively.

Here we are going to prove the following theorem:

Theorem 1 Let $\mathbf{a}_{0} \leq_{\omega} \mathbf{a}_{1} \leq_{\omega} \cdots \leq_{\omega} \mathbf{a}_{n} \leq_{\omega} \ldots$ be an increasing sequence in $\mathcal{D}_{\omega}$. Then for each $\mathbf{g} \in \mathcal{D}_{\omega}$, such that $\mathbf{a}_{n} \leq_{\omega} \mathbf{g}$, for all $n$, there is an $\mathbf{f} \in \mathcal{D}_{1}$ such that $\mathbf{f}, \mathbf{g}$ form an exact pair for the sequence $\left\{\mathbf{a}_{n}\right\}_{n<\omega}$. Even more. For arbitrary $k$ the degrees $\mathbf{f}^{(k)}, \mathbf{g}^{(k)}$ form an exact pair for the sequence $\left\{\mathbf{a}_{n}^{(k)}\right\}_{n<\omega}$, where by $\mathbf{x}^{(k)}$ we denote the $k$-th jump of the degree $\mathbf{x}$ in $\mathcal{D}_{\omega}$.

As a corollary we obtain the following exact pair theorem for the enumeration degrees.

Theorem 2 Let $\mathbf{a}_{0} \leq_{e} \mathbf{a}_{1} \leq_{e} \cdots \leq_{e} \mathbf{a}_{n} \leq_{e} \ldots$ be an increasing sequence in the semi-lattice of the enumeration degrees $\mathcal{D}_{e}$. Then for each $\mathbf{g} \in \mathcal{D}_{e}$, such that $\mathbf{a}_{n} \leq_{e} \mathbf{g}$, for all $n$, there is an $\mathbf{f} \in \mathcal{D}_{e}$ such that $\mathbf{f}^{(k)}, \mathbf{g}^{(k)}$ form an exact pair for the sequence $\left\{\mathbf{a}_{n}^{(k)}\right\}_{n<\omega}$ for arbitrary $k$, where by $\mathbf{x}^{(k)}$ we denote the $k$-th enumeration jump of the degree $\mathbf{x}$.

\section{Preliminaries}

Let $W_{0}, W_{1}, \ldots, W_{n}, \ldots$ be a Gödel enumeration of the recursively enumerable sets. We will use the same notation for the enumeration operators, i.e., the operators over sets of natural numbers acting by the rule $W_{e}(A)=\{x \mid \exists v(\langle x, v\rangle \in$ $\left.\left.W_{e} \& D_{v} \subseteq A\right)\right\}$ for each $A \subseteq \mathbf{N}$, where by $D_{v}$ we shall denote the finite set with canonical index $v$.

The enumeration jump of a set $A$ is defined as $A^{\prime}=L_{A} \oplus \mathbf{N} \backslash L_{A}$, where $L_{A}=\left\{\langle a, x\rangle \mid x \in W_{a}(A)\right\}$.

Given a sequence $\mathcal{A}=\left\{A^{n}\right\}_{n<\omega}$ we define its jump sequence $P(\mathcal{A})=\left\{P^{n}(\mathcal{A})\right\}_{n<\omega}$ as follows:

$$
\begin{aligned}
& \text { (i) } & P^{0}(\mathcal{A}) & =A^{0} \\
& \text { (ii) } & P^{n+1}(\mathcal{A}) & =P^{n}(\mathcal{A})^{\prime} \oplus A^{n+1} .
\end{aligned}
$$

In [3] it is shown that for any two sequences $\mathcal{A}, \mathcal{B}$ we have that $\mathcal{A} \leq_{u} \mathcal{B} \Longleftrightarrow$ there is a recursive function $g$, such that $A^{n}=W_{g(n)}\left(P^{n}(\mathcal{B})\right)$ for all natural $n$ (in particular $A^{n} \leq_{e} P^{n}(B)$ ). This gives us that $\mathcal{A} \equiv_{u} P(\mathcal{A})$. The jump of a sequence $\mathcal{A} \equiv_{u}\left\{P^{n}(\mathcal{A})\right\}_{n<\omega}$ is the sequence $\mathcal{A}^{\prime}=\left\{P^{n+1}(\mathcal{A})\right\}_{n<\omega}$. It is true that $J_{\mathcal{A}^{\prime}}=\left\{\mathbf{a}^{\prime} \mid \mathbf{a} \in J_{\mathcal{A}}\right\}$ and that the jump agrees with the embedding of the $e$-degrees, so $(A, \emptyset, \emptyset, \ldots, \emptyset)^{\prime} \equiv_{u}\left(A^{\prime}, \emptyset, \emptyset, \ldots, \emptyset\right)$.

Now we are ready to show that Theorem 2 follows from Theorem 1 . 
Proof of Theorem 2. Suppose that we have an increasing sequence of enumeration degrees $\mathbf{a}_{0} \leq_{e} \mathbf{a}_{1} \leq_{e} \cdots \leq_{e} \mathbf{a}_{n} \leq_{e} \ldots$ and let $\mathbf{g}$ be an enumeration degree, such that $\mathbf{g}$ is an upper bound for $\left\{\mathbf{a}_{n}\right\}_{n<\omega}$. Fix a sequence of sets $A_{0}, A_{1}, \ldots, A_{n}, \ldots$ such that $\mathbf{a}_{n}=d_{e}\left(A_{n}\right)$ and let $G$ be such that $\mathbf{g}=d_{e}(G)$. Now for each $n$ we take $\mathcal{A}_{n}$ to be the sequence $\left(A_{n}, \emptyset, \emptyset, \ldots, \emptyset, \ldots\right)$ and $\mathcal{G}$ to be the sequence $(G, \emptyset, \emptyset, \ldots, \emptyset, \ldots)$. It is clear that $\mathcal{A}_{0} \leq_{u} \mathcal{A}_{1} \leq_{u} \cdots \leq_{u} \mathcal{A}_{n} \leq_{u} \cdots \leq_{u} \mathcal{G}$. Thus we have an increasing sequence

$$
d_{\omega}\left(\mathcal{A}_{0}\right) \leq_{\omega} d_{\omega}\left(\mathcal{A}_{1}\right) \leq_{\omega} \cdots \leq_{\omega} d_{\omega}\left(\mathcal{A}_{n}\right) \leq_{\omega} \cdots \leq_{\omega} d_{\omega}(\mathcal{G})
$$

of $\omega$-enumeration degrees.

Now according to Theorem 1 there is an $\omega$-enumeration degree $\mathbf{f} \in \mathcal{D}_{1}$, such that $\mathbf{f}$ and $d_{\omega}(\mathcal{G})$ form an exact pair for the sequence $\left\{d_{\omega}\left(\mathcal{A}_{n}\right)\right\}_{n<\omega}$. Since $\mathbf{f} \in \mathcal{D}_{1}$, then $\mathbf{f}$ is of the form $\mathbf{f}=d_{\omega}(\mathcal{F})$, where $\mathcal{F}=(F, \emptyset, \emptyset, \ldots, \emptyset, \ldots)$. We claim that $d_{e}(G)$ and $d_{e}(F)$ form an exact pair for the sequence $\mathbf{a}_{0} \leq_{e} \mathbf{a}_{1} \leq_{e}$ $\cdots \leq_{e} \mathbf{a}_{n} \leq_{e} \ldots$ Indeed: suppose that some enumeration degree $\mathbf{x}$ has the property $\mathbf{x} \leq d_{e}(G)$ and $\mathbf{x} \leq d_{e}(F)$. Let $\mathbf{x}=d_{e}(X)$ and consider the sequence $\mathcal{X}=(X, \emptyset, \emptyset, \ldots, \emptyset, \ldots)$. We have that $\mathcal{X} \leq_{u} \mathcal{F}$ and $\mathcal{X} \leq_{u} \mathcal{G}$ and therefor $\mathcal{X} \leq_{u} \mathcal{A}_{n}$ for some $n$. But then $X \leq_{e} A_{n}$, which proves that $d_{e}(F)$ and $d_{e}(G)$ are an exact pair for the sequence $\mathbf{a}_{0} \leq_{e} \mathbf{a}_{1} \leq_{e} \cdots \leq_{e} \mathbf{a}_{n} \leq_{e} \cdots$

According to the definition of the jump operation in $\mathcal{D}_{\omega}$ we have that the sequences $\mathcal{A}_{n}^{\prime}, \mathcal{G}^{\prime}$ and $\mathcal{F}^{\prime}$ are equivalent to the sequences $\left(A_{n}^{\prime}, \emptyset, \emptyset, \ldots, \emptyset, \ldots\right)$, $\left(G^{\prime}, \emptyset, \emptyset, \ldots, \emptyset, \ldots\right)$ and $\left(F^{\prime}, \emptyset, \emptyset, \ldots, \emptyset, \ldots\right)$ respectively. Applying the same reasoning as above, we obtain that $d_{e}\left(F^{\prime}\right)$ and $d_{e}\left(G^{\prime}\right)$ (which are actually $d_{e}(F)^{\prime}$ and $\left.d_{e}(G)^{\prime}\right)$ are an exact pair for the sequence $\mathbf{a}_{0}^{\prime} \leq_{e} \mathbf{a}_{1}^{\prime} \leq_{e} \cdots \leq_{e} \mathbf{a}_{n}^{\prime} \leq_{e} \cdots$

In the rest of the paper we shall present a proof of Theorem 1.

\section{Proof of Theorem 1}

First we will prove the following omitting theorem:

Theorem 3 Let $\mathcal{A}_{0} \leq_{u} \mathcal{A}_{1} \leq_{u} \ldots \leq_{u} \mathcal{A}_{n} \ldots$ be an increasing sequence of sequences of sets of natural numbers. Let also $\left\{\mathcal{R}_{k}\right\}_{k<\omega}$ be a sequence of elements of $\mathcal{S}$ such that $\mathcal{R}_{k} \mathbb{Z}_{u} \mathcal{A}_{n}$, for each $k$ and $n$. Then there is an $\mathcal{F} \in \mathcal{S}$ such that $\mathcal{A}_{n} \leq_{u} \mathcal{F}$, for each $n$, but $\mathcal{R}_{k} \mathbb{\leq}_{u} \mathcal{F}$ for all $k$.

For simplicity we will show how to omit only one sequence $\mathcal{R}$. However, by a simple and standard modification we can use the same technique to omit countably many sequences.

Let us a fix an increasing sequence $\mathcal{A}_{0} \leq_{u} \mathcal{A}_{1} \leq_{u} \cdots \leq_{u} \mathcal{A}_{n} \ldots$ of elements of $\mathcal{S}$ and let $\mathcal{R} \in \mathcal{S}$ be such that $\mathcal{R} \not_{u} \mathcal{A}_{n}$, for every natural $n$. We will suppose that $\mathcal{A}_{n}=\left\{A_{n}^{k}\right\}_{k<\omega}$ and $\mathcal{R}=\left\{R^{k}\right\}_{k<\omega}$ (we will use upper indexes for coordinates in the rest of the paper). Fix a sequence $g_{0}, g_{1}, \ldots, g_{n}, \ldots$ of all recursive functions.

We will search $\mathcal{F}$ in the form $\mathcal{F}=\left(f^{0}, f^{1}, \ldots, f^{n}, \ldots\right)$, where $f^{n}$ is a mapping from $\mathbf{N}$ in $\mathbf{N}$. We will use the notation $\vec{f}$ instead of $\mathcal{F}$. We will construct $\mathcal{F}$ by 
using a forcing technique so we have to define the modelling and forcing relations and the notion of finite parts.

Let us begin with the "finite" parts. We shall call finite part every sequence of the form $\vec{\tau}=\left(\tau^{0}, \tau^{1}, \ldots, \tau^{n}, \ldots\right)$, where each $\tau^{i}$ is a mapping from of an initial segment of $\mathbf{N}$ into $\mathbf{N}$. By $\operatorname{lh}\left(\tau^{i}\right)$ we shall denote the length of the initial segment which is the domain of $\tau^{i}$. We shall use the notation $\vec{\tau}, \vec{\rho}, \vec{\delta}$ and $\vec{\sigma}$ (sometimes with indexes) for finite parts and $\tau^{i}, \rho^{i}, \delta^{i}$ and $\sigma^{i}$ for the respective coordinates.

If $n$ is a natural number, by $\vec{n}$ we shall denote an increasing sequence $a_{0}<$ $a_{1}<a_{2}<\ldots<a_{n}$ of $n+1$ natural numbers. We will write $\vec{n} \preceq \vec{m}$ if $n \leq m$ and $\vec{m}$ is an extension of $\vec{n}$.

Since $\vec{f}$ has to "encode" in itself all sequences $\mathcal{A}_{n}$ we will regard only special finite parts which we will call $\vec{n}$-coding finite parts and which are defined by:

Definition 2 If $\vec{n}=a_{0}<a_{1}<a_{2}<\ldots<a_{n}$ is an increasing sequence of natural numbers and $\vec{\tau}$ is a finite part, we say that $\vec{\tau}$ is $\vec{n}$-coding iff for all $i$ we have that:

$$
a_{k}<\langle x, k\rangle<\operatorname{lh}\left(\tau^{i}\right) \Longrightarrow \tau^{i}(\langle x, k\rangle) \in A_{k}^{i}
$$

Now we are ready to define the modelling and forcing relations for the conditions $F_{e}^{k}$ (the condition $F_{e}^{k}$ will be responsible for what happens to the $k$-th coordinate when we apply the enumeration operator $W_{e}$ to it). We begin with the modeling relation since it is more natural.

Definition 3 Let $\vec{f}=\left(f^{0}, f^{1}, \ldots, f^{n}, \ldots\right)$ be a sequence of mappings from $\mathbf{N}$ into $\mathbf{N}$. We define the relations $\vec{f}=(\neg) F_{e}^{k}(x)$ (for each e) using induction on $k$ :

(i) $\vec{f} \mid=F_{e}^{0}(x) \Longleftrightarrow x \in W_{e}\left(\operatorname{Graph}\left(f^{0}\right)\right)$.

(ii) $\vec{f}=\neg F_{e}^{0}(x) \Longleftrightarrow \vec{f} \not F_{e}^{0}(x)$.

(iii) $\vec{f}=F_{e}^{k+1}(x)$ if there exist $u, u_{0}$ and $u_{1}$ such that

(1) $\langle x, u\rangle \in W_{e}$;

(2) $D_{u}=D_{u_{1}} \oplus D_{u_{2}}$;

(3) for each $v \in D_{u_{1}}$ is either true that $v=2\left\langle e_{v}, x_{v}\right\rangle$ and $\vec{f}=F_{e_{v}}^{k}\left(x_{v}\right)$, or $v=2\left\langle e_{v}, x_{v}\right\rangle+1$ and $\vec{f} \models \neg F_{e_{v}}^{k}\left(x_{v}\right)$;

(4) $D_{u_{2}} \subseteq$ Graph $f^{k+1}$.

(iv) $\vec{f}=\neg F_{e}^{k+1}(x) \Longleftrightarrow \vec{f} \not F_{e}^{k+1}(x)$.

Note that from the definition it follows that do determine whether $\vec{f}$ $(\neg) F_{e}^{k}(x)$ it is sufficient to know only the first $k+1$ elements of the sequence $\vec{f}$, i.e., $f^{0}, f^{1}, \ldots, f^{k}$. Even more. The following proposition is true:

Proposition 1 Let $\vec{f}$ be a sequence of mappings from $\mathbf{N}$ in $\mathbf{N}$. Then for each $e$ and each $k$

$$
\vec{f}=F_{e}^{k}(x) \Longleftrightarrow x \in W_{e}\left(P^{k}(\vec{f})\right)
$$


Now we will give the definition of the forcing relation.

Definition 4 Let $\vec{n}$ be an increasing sequence of $n+1$ natural numbers and let $\vec{\tau}$ be an $\vec{n}$-coding sequence of finite parts. We define the relations $\vec{\tau} \Vdash \vec{n}$ $(\neg) F_{e}^{k}(x)$ (for each $e$ ) using induction on $k$ :

(i) $\vec{\tau} \Vdash{ }_{\vec{n}} F_{e}^{0}(x) \Longleftrightarrow x \in W_{e}\left(\operatorname{Graph} \tau^{0}\right)$.

(ii) $\vec{\tau} \Vdash \vec{n} \neg F_{e}^{0}(x) \Longleftrightarrow(\forall \vec{\rho} \supseteq \tau)\left(\vec{\rho}\right.$ is $\vec{n}$-coding $\left.\Rightarrow \vec{\rho} \forall \vec{n} F_{e}^{0}(x)\right)$.

(iii) $\vec{\tau} \Vdash \vec{n}_{\vec{n}} F_{e}^{k+1}$ if there exist $u, u_{0}$ and $u_{1}$ such that:

(1) $\langle x, u\rangle \in W_{e}$;

(2) $D_{u}=D_{u_{1}} \oplus D_{u_{2}}$;

(3) for each $v \in D_{u_{1}}$ is either true that $v=2\left\langle e_{v}, x_{v}\right\rangle$ and $\vec{\tau} \Vdash \Vdash_{\vec{n}} F_{e_{v}}^{k}\left(x_{v}\right)$, or $v=2\left\langle e_{v}, x_{v}\right\rangle+1$ and $\vec{\tau} \Vdash \vec{n} \neg F_{e_{v}}^{k}\left(x_{v}\right)$;

(4) $D_{u_{2}} \subseteq$ Graph $\tau^{k+1}$.

(iv) $\vec{\tau} \Vdash_{\vec{n}} \neg F_{e}^{k+1}(x) \Longleftrightarrow(\forall \vec{\rho} \supseteq \tau)\left(\vec{\rho}\right.$ is $\vec{n}$-coding $\left.\Rightarrow \vec{\rho} \forall \vec{n} F_{e}^{k+1}(x)\right)$.

Again from the definition it follows that the relations $\vec{\tau} \Vdash_{\vec{n}}(\neg) F_{e}^{k}(x)$ depend only on the first $k+1$ members of the sequence $\vec{\tau}$.

The next proposition shows that the forcing relations are monotone.

Proposition 2 Let $\vec{m}$ and $\vec{n}$ be two increasing sequences of natural numbers of length $m+1$ and $n+1$ respectively. Let also $\vec{\tau}$ and $\vec{\rho}$ be two finite parts. Then the following are true:

(1) if $\vec{\tau}$ and $\vec{\rho}$ are both $\vec{n}$-coding and $\vec{\tau} \subseteq \vec{\rho}$, then

$$
\vec{\tau} \Vdash_{\vec{n}}(\neg) F_{e}^{k}(x) \Longrightarrow \vec{\rho} \Vdash_{\vec{n}}(\neg) F_{e}^{k}(x) ;
$$

(2) if $\vec{m} \preccurlyeq \vec{n}$ and $\vec{\tau}$ is $\vec{n}$-coding, then $\vec{\tau}$ is $\vec{m}$-coding and

$$
\vec{\tau} \Vdash_{\vec{m}}(\neg) F_{e}^{k}(x) \Longrightarrow \vec{\tau} \Vdash_{\vec{n}}(\neg) F_{e}^{k}(x) ;
$$

(3) if $\vec{m} \preccurlyeq \vec{n}$ and $\vec{\tau}$ is $\vec{m}$-coding, $\vec{\rho}$ is $\vec{n}$-coding and $\vec{\tau} \subseteq \vec{\rho}$, then

$$
\vec{\tau} \Vdash_{\vec{m}}(\neg) F_{e}^{k}(x) \Longrightarrow \vec{\rho} \Vdash_{\vec{n}}(\neg) F_{e}^{k}(x) .
$$

Now we are ready to begin the construction. The construction of $\vec{f}$ will be carried out by steps. At each step $s$ we will construct a finite part $\vec{\tau}_{s}$ and a monotone sequence $\vec{s}$ in such a way that $\vec{\tau}_{s}$ is $\vec{s}$-coding and also that $\vec{\tau}_{s} \subseteq \vec{\tau}_{s+1}$ and $\vec{s} \preccurlyeq \overrightarrow{s+1}$. Finally we will set $\vec{f}=\bigcup_{s<\omega} \vec{\tau}_{s}$ and we will obtain an infinite increasing sequence $\vec{\omega}=\bigcup_{s<\omega} \vec{s}$ of natural numbers.

At step 0 we set $\vec{\tau}_{0}=(\emptyset, \emptyset, \ldots, \emptyset, \ldots)$ and $\overrightarrow{0}=0$.

Now suppose that $\vec{\tau}_{s}$ and $\vec{s}$ are defined. We divide the step $s$ into three substeps.

First substep. We build an $\vec{s}$-coding finite part $\vec{\delta}_{s}$ such that $\vec{\tau}_{s} \subseteq \vec{\delta}_{s}$ and $\vec{\delta}_{s}$ encodes the first elements of sets $A_{i}^{j}$, for $i, j \leq s$, which are not encoded by $\vec{\tau}_{s}$. 
Second substep. We build an $\vec{s}$-coding finite part $\vec{\sigma}_{s} \supseteq \vec{\delta}_{s}$ such that for each $k \leq s$ and each $\langle e, x\rangle \leq s$ either $\vec{\sigma} \Vdash \vec{s} F_{e}^{k}(x)$ or $\vec{\sigma} \Vdash{ }_{s} \neg F_{e}^{k}(x)$.

Third substep. We consider the sequence $\left\{C_{s}^{n}\right\}_{n<\omega}$, where

$C_{s}^{n}=\left\{x \mid\left(\exists \vec{\rho} \supseteq^{n} \vec{\sigma}_{s}\right)\left(\vec{\rho}\right.\right.$ is $\vec{s}$-coding \& $\left.\left.\rho^{0}\left(\operatorname{lh} \sigma_{s}^{0}\right) \simeq x \& \vec{\rho} \Vdash \vec{s}_{s} F_{g_{s}(n)}^{n}\left(\operatorname{lh} \sigma_{s}^{0}\right)\right)\right\}$,

where $\vec{\rho} \supseteq^{n} \vec{\sigma}_{s}$ means that $\rho^{i} \supseteq \sigma_{s}^{i}$ for $0 \leq i \leq n$, and $\rho^{i}=\sigma_{s}^{i}$ for $i>n$. The sequence $\left\{C_{s}^{n}\right\}_{n<\omega}$ is uniformly reducible to the sequence $\mathcal{A}_{s}$, i.e., $\left\{C_{s}^{n}\right\}_{n<\omega} \leq u$ $\mathcal{A}_{s}$ and therefore $\left\{C_{s}^{n}\right\}_{n<\omega} \neq \mathcal{R}$ (for a detailed proof of an analogous statement see [3]). Let $n$ be such that $C_{s}^{n} \neq R_{n}$. Then there is a $x$ such that either $x \in$ $C_{s}^{n} \& x \notin R_{n}$ or $x \notin C_{s}^{n} \& x \in R_{n}$.

If $x \in C_{s}^{n} \& x \notin R_{n}$ is the case, we take one $\vec{\rho} \supseteq^{n} \vec{\sigma}_{s}$ such that $\vec{\rho}$ is $\vec{s}$-coding, $\rho^{0}\left(\operatorname{lh} \sigma_{s}^{0}\right) \simeq x$ and $\vec{\rho} \Vdash \vec{s}_{s} F_{g_{s}(n)}^{n}\left(\operatorname{lh} \sigma_{s}^{0}\right)$, and we set $\vec{\tau}_{s+1}=\vec{\rho}$.

If $x \notin C_{s}^{n} \& x \in R_{n}$ then we set $\vec{\tau}_{s+1}$ to be an $\vec{s}$-coding finite part such that $\tau_{s+1}^{0}\left(\operatorname{lh} \sigma_{s}^{0}\right) \simeq x$. Note that in this case is true that

$$
\left(\forall \vec{\rho} \supseteq^{n} \vec{\tau}_{s+1}\right)\left(\vec{\rho} \text { is } \vec{s} \text {-coding } \Longrightarrow \vec{\rho} \forall \vec{s}_{g_{s}(n)}\left(\operatorname{lh} \sigma_{s}^{0}\right)\right) .
$$

Since the forcing relation $\vec{\rho} \Vdash \vec{s}_{s} F_{g_{s}(n)}^{n}$ depends only on the first $n+1$ elements of $\vec{\rho}$, we have that

$$
\left(\forall \vec{\rho} \supseteq \vec{\tau}_{s+1}\right)\left(\vec{\rho} \text { is } \vec{s} \text {-coding } \Longrightarrow \vec{\rho} \forall \vec{s}_{g_{s}(n)} \mathcal{F}_{g_{s}}^{n}\left(\operatorname{lh} \sigma_{s}^{0}\right)\right)
$$

which means that $\vec{\tau}_{s+1} \Vdash \vec{s}_{s} \neg F_{g_{s}(i)}^{n}\left(\operatorname{lh} \sigma_{s}^{0}\right)$.

Finally we set $\overrightarrow{s+1}=\vec{s} * \max \left\{\operatorname{lh} \tau_{s+1}^{i} \mid i<\omega\right\}$, which concludes the construction. In order to complete the proof, we have to prove three properties of $\vec{f}$.

Claim $1 \mathcal{A}_{n} \leq_{u} \vec{f}$, for each $n$.

Proof. Let $\vec{\omega}=a_{0}, a_{1}, \ldots, a_{i}, \ldots$. Then, according to the first substep of each step of the construction, we have that $A_{n}^{i}=\left\{f_{i}(\langle x, i\rangle) \mid\langle x, i\rangle \geq a_{n}\right\}$.

Claim 2 (Truth lemma) For each $e$ and each $k$

$$
\vec{f} \models(\neg) F_{e}^{k}(x) \Leftrightarrow(\exists \vec{m} \preccurlyeq \vec{\omega})(\exists \vec{\tau} \subseteq \vec{f})\left(\vec{\tau} \text { is } \vec{m} \text {-coding \& } \vec{\tau} \Vdash_{\vec{m}}(\neg) F_{e}^{k}(x)\right) \text {. }
$$

Proof. This property is assured from the second substeps. We will prove it using induction on $k$. First consider $k=0$. The positive equivalence is obvious from the definition of the relations $\models F_{e}^{0}$ and $\Vdash \vec{m} F_{e}^{0}$. Now we will prove the equivalence

$$
\vec{f} \models \neg F_{e}^{0}(x) \Leftrightarrow(\exists \vec{m} \preccurlyeq \vec{\omega})(\exists \vec{\tau} \subseteq \vec{f})\left(\vec{\tau} \text { is } \vec{m} \text {-coding \& } \vec{\tau} \Vdash \vec{m} \neg F_{e}^{0}(x)\right) .
$$

The right to left direction follows from the positive equivalence. Let us prove the left to right direction. Suppose that $\vec{f} \models \neg F_{e}^{0}(x)$. Then consider a step 
$s+1$ such that $s\rangle\langle e, x\rangle$. In the second substep we have constructed an $\vec{s}$ coding finite part $\vec{\sigma}_{s}$ such that either $\vec{\sigma}_{s} \Vdash_{\vec{s}} F_{e}^{0}(x)$ or $\vec{\sigma}_{s} \Vdash_{\vec{s}} \neg F_{e}^{0}(x)$ and $\vec{\sigma}_{s} \subseteq \vec{\tau}_{s+1} \subseteq \vec{f}$. If $\vec{\sigma}_{s} \Vdash_{s} F_{e}^{0}(x)$ than from the positive equivalence we obtain that $\vec{f} \models F_{e}^{0}(x)$, which contradicts $\vec{f} \models F_{e}^{0}(x)$. Therefore $\vec{\sigma}_{s} \Vdash \vec{s}_{\vec{s}} \neg F_{e}^{0}(x)$.

Now suppose that the statement is true for $k$. First we prove that

$$
\vec{f} \models F_{e}^{k+1}(x) \Leftrightarrow(\exists \vec{m} \preccurlyeq \vec{\omega})(\exists \vec{\tau} \subseteq \vec{f})\left(\vec{\tau} \text { is } \vec{m} \text {-coding \& } \vec{\tau} \Vdash_{\vec{m}} F_{e}^{k+1}(x)\right) .
$$

For the left to right direction suppose that $\vec{f} \models F_{e}^{k+1}(x)$. Then, according to the definition we have that, there are $u, u_{1}$ and $u_{2}$ such that:

(1) $\langle x, u\rangle \in W_{e}$;

(2) $D_{u}=D_{u_{1}} \oplus D_{u_{2}}$

(3) for all $v \in D_{u_{1}}$ is either true that $v=2\left\langle e_{v}, x_{v}\right\rangle$ and $\vec{f} \models F_{e_{v}}^{k}\left(x_{v}\right)$, or $v=2\left\langle e_{v}, x_{v}\right\rangle+1$ and $\vec{f}=\neg F_{e_{v}}^{k}\left(x_{v}\right)$.

(4) $D_{u_{2}} \subseteq$ Graph $f_{k+1}$

Consider (3). According to the induction hypothesis, for each $v \in D_{u_{1}}$ we can find $\vec{m}_{v} \preccurlyeq \vec{\omega}$ and $\vec{\rho}_{v} \subseteq \vec{f}$, such that $\vec{\rho}_{v}$ is $\vec{m}_{v}$-coding and

$$
\begin{gathered}
v=2\left\langle e_{v}, x_{v}\right\rangle \Longrightarrow \vec{\rho}_{v} \Vdash_{\vec{m}_{v}} F_{e_{v}}^{k}\left(x_{v}\right), \\
v=2\left\langle e_{v}, x_{v}\right\rangle+1 \Longrightarrow \vec{\rho}_{v} \Vdash_{\vec{m}_{v}} \neg F_{e_{v}}^{k}\left(x_{v}\right) .
\end{gathered}
$$

Since $\vec{m}_{v} \preccurlyeq \vec{\omega}$ and $\vec{\rho}_{v} \subseteq \vec{f}$, there is an $\vec{m} \preccurlyeq \vec{\omega}$ and a $\vec{\tau} \subseteq \vec{f}$, such that for each $v, \vec{m}_{v} \preccurlyeq \vec{m}$ and $\vec{\rho}_{v} \subseteq \vec{\tau}$. Then from the monotonicity of the forcing relation we obtain that for each $v \in D_{u_{1}}$

$$
\begin{gathered}
v=2\left\langle e_{v}, x_{v}\right\rangle \Longrightarrow \vec{\tau} \Vdash_{\vec{m}} F_{e_{v}}^{k}\left(x_{v}\right), \\
v=2\left\langle e_{v}, x_{v}\right\rangle+1 \Longrightarrow \vec{\tau} \Vdash_{\vec{m}} \neg F_{e_{v}}^{k}\left(x_{v}\right),
\end{gathered}
$$

which is exactly point (3) from the definition of the forcing relation $\Vdash_{\vec{m}} F_{e}^{k+1}$. We can extend the $k+2$-nd element of $\vec{\tau}$ in such a way that $\tau^{k+1} \subseteq \vec{f}_{k+1}$ and $D_{u_{2}} \subseteq$ Graph $\tau_{k+1}$. Of course this extension does not afflict the forcing relations that we have satisfied, since they depend only on the first $k+1$ elements of $\vec{\tau}$.

Thus we obtain that $\vec{\tau} \subseteq \vec{f}, \vec{\tau}$ is $\vec{m}$-coding and $\vec{\tau} \Vdash F_{e}^{k+1}(x)$.

For the opposite direction, suppose that there is an $\vec{m} \preccurlyeq \vec{\omega}$ and a $\vec{\tau} \subseteq \vec{f}$ such that $\vec{\tau}$ is $\vec{m}$-coding and $\vec{\tau} \Vdash \Vdash_{\vec{m}} F_{e}^{k+1}(x)$. Then we have, that there are $u$, $u_{1}$ and $u_{2}$ such that

(1) $\langle x, u\rangle \in W_{e}$;

(2) $D_{u}=D_{u_{1}} \oplus D_{u_{2}}$;

(3) for all $v \in D_{u_{1}}$ either $v=2\left\langle e_{v}, x_{v}\right\rangle$ and $\vec{\tau} \Vdash_{\vec{m}} F_{e_{v}}^{k}\left(x_{v}\right)$, or $v=2\left\langle e_{v}, x_{v}\right\rangle+1$ and $\vec{\tau} \Vdash_{\vec{m}} \neg F_{e_{v}}^{k}\left(x_{v}\right)$.

(4) $D_{u_{2}} \subseteq$ Graph $\tau_{k+1}$.

Applying the induction hypothesis to (3) we obtain

(1) $\langle x, u\rangle \in W_{e}$;

(2) $D_{u}=D_{u_{1}} \oplus D_{u_{2}}$; 
(3) for all $v \in D_{u_{1}}$ either $v=2\left\langle e_{v}, x_{v}\right\rangle$ and $\vec{f} \models F_{e_{v}}^{k}\left(x_{v}\right)$, or $v=2\left\langle e_{v}, x_{v}\right\rangle+1$ and $\vec{f} \models \neg F_{e_{v}}^{k}\left(x_{v}\right)$;

(4) $D_{u_{2}} \subseteq$ Graph $\tau_{k+1} \subseteq f_{k+1}$,

which is exactly what we have to prove. This concludes the proof of the positive equivalence for $k+1$. The proof of the negative equivalence is analogous to that for $k=0$.

Claim $3 \mathcal{R} \not_{u} \vec{f}$.

Proof. In order to obtain a contradiction assume that $\mathcal{R} \leq_{u} \vec{f}$. Then the sequence $\left\{f^{0^{-1}}\left(R_{n}\right)\right\}_{n<\omega}$ is also uniformly reducible to $\vec{f}$. Therefore there is a recursive function $g_{s}$ such that $f^{0^{-1}}\left(R_{n}\right)=W_{g_{s}(n)}\left(P^{n}(\vec{f})\right)$ for each $n$, which means

$$
x \in f^{0^{-1}}\left(R_{n}\right) \Longleftrightarrow \vec{f} \models F_{g_{s}(n)}^{n}(x)
$$

Now consider the third substep of the $s+1$-st step. There we have constructed a finite part $\vec{\tau}_{s+1}$ extending the finite part $\vec{\sigma}_{s}$ such that for some $i$

$$
\vec{\tau}_{s+1} \Vdash{ }_{s}(\neg) F_{g_{s}(i)}^{i}\left(\operatorname{lh} \sigma_{s}^{0}\right) \Longleftrightarrow(\neg)\left(\tau_{s+1}^{0}\left(\mathrm{lh} \sigma_{s}^{0}\right) \notin R_{i}\right) .
$$

Now using the Truth Lemma and that $\tau_{s+1}^{0} \subseteq f^{0}$ we obtain that

$$
\begin{gathered}
\operatorname{lh} \sigma_{s}^{0} \in f^{0^{-1}}\left(R_{i}\right) \Rightarrow f^{0}\left(\operatorname{lh} \sigma_{s}^{0}\right) \in R_{i} \Rightarrow \neg\left(f^{0}\left(\operatorname{lh} \sigma_{s}^{0}\right) \notin R_{i}\right) \Rightarrow \vec{f} \models \neg F_{g_{s}(i)}^{i}\left(\operatorname{lh} \sigma_{s}^{0}\right) ; \\
\operatorname{lh} \sigma_{s}^{0} \notin f^{0^{-1}}\left(R_{i}\right) \Rightarrow f^{0}\left(\operatorname{lh} \sigma_{s}^{0}\right) \notin R_{i} \Rightarrow \vec{f} \models F_{g_{s}(i)}^{i}\left(\operatorname{lh} \sigma_{s}^{0}\right),
\end{gathered}
$$

which is a contradiction with $x \in f^{0^{-1}\left(R_{n}\right)} \Longleftrightarrow \vec{f} \models F_{g_{s}(n)}^{n}(x)$. This means that the assumption $\mathcal{R} \leq_{u} \vec{f}$ leads to a contradiction and therefore we have that $\mathcal{R} \mathbb{L}_{u} \vec{f}$.

Claim 1 and Claim 3 are exactly the properties of $\vec{f}$ that we had to prove in order to prove Theorem 3 and so this concludes its proof.

We are ready to prove Theorem 1.

Proof of Theorem 1. Let $\mathbf{a}_{1} \leq_{\omega} \mathbf{a}_{2} \leq_{\omega} \cdots \leq_{\omega} \mathbf{a}_{n} \leq_{\omega} \cdots \leq_{\omega} \mathrm{g}$ be an infinite increasing sequence of $\omega$-enumeration degrees. Fix an increasing sequence $\mathcal{A}_{1} \leq_{u} \mathcal{A}_{2} \leq_{u} \cdots \leq_{u} \mathcal{A}_{n} \leq_{u} \cdots \leq_{u} \mathcal{G}$ of elements of $\mathcal{S}$ such that $\mathbf{a}_{n}=d_{\omega}\left(\mathcal{A}_{n}\right)$ and $\mathbf{g}=d_{\omega}(\mathcal{G})$. Since there are only countably many sequences $u$-reducible to $\mathcal{G}$ then the set $\left\{\mathcal{X} \in \mathcal{S} \mid \mathcal{X} \leq_{u} \mathcal{G} \& \forall n\left(\mathcal{X} \mathbb{Z}_{u} \mathcal{A}_{n}\right)\right\}$ is denumerable. Let us order its elements into the sequence $\left\{\mathcal{R}_{k}\right\}_{k<\omega}$. Now it is clear that if we take an $\mathcal{F}^{*} \in \mathcal{S}$ as in Theorem 3, then $d_{\omega}\left(\mathcal{F}^{*}\right)$ and $d_{\omega}(\mathcal{G})$ will be an exact pair for the sequence $\left\{\mathbf{a}_{n}\right\}_{n<\omega}$.

Now in order to obtain an $\mathbf{f} \in \mathcal{D}_{1}$, such that $\mathbf{g}$, $\mathbf{f}$ form an exact pair for the sequence $\left\{\mathbf{a}_{k}\right\}_{k<\omega}$, we have to use the omitting theorem from [3]: 
Theorem 4 (Soskov, Kovachev) Let $\mathcal{A} \in \mathcal{S}$ and let $\left\{\mathcal{R}_{k}\right\}_{k<\omega}$ be a sequence of elements of $\mathcal{S}$, such that $\mathcal{R}_{k} \mathbb{Z}_{u} \mathcal{A}$. Then there is a sequence $\mathcal{F}=(F, \emptyset, \emptyset, \ldots$, $\emptyset, \ldots)$, such that $\mathcal{A} \leq_{u} \mathcal{F}$ and $\mathcal{R}_{k} \mathbb{Z}_{u} \mathcal{F}$, for all $k$.

Therefore we can take a sequence $\mathcal{F}=(F, \emptyset, \emptyset, \ldots, \emptyset, \ldots)$, such that $\mathcal{F}^{*} \leq_{u} \mathcal{F}$ and $\mathcal{F}$ omits all the sequences which are under $\mathcal{G}$, but are not under $\mathcal{F}^{*}$. Now set $\mathbf{f}=d_{\omega}(\mathcal{F})$. It is clear that $\mathbf{f} \in \mathcal{D}_{1}$ and that $\mathbf{f}$ and $\mathbf{g}$ form an exact pair for the sequence $\left\{\mathbf{a}_{n}\right\}_{n<\omega}$.

For the last part of the theorem is sufficient to prove the following

Proposition 3 Let $\mathbf{f}, \mathbf{g} \in \mathcal{D}_{\omega}$ form an exact pair for the increasing sequence $\left\{\mathbf{a}_{n}\right\}_{n<\omega}$ of $\omega$-enumeration degrees. Then $\mathbf{f}^{\prime}$ and $\mathbf{g}^{\prime}$ form an exact pair for the sequence $\left\{\mathbf{a}_{n}^{\prime}\right\}_{n<\omega}$

Proof. Let $\mathbf{f}=d_{\omega}(\mathcal{F})$ and $\mathbf{g}=d_{\omega}(\mathcal{G})$. Then from the definition of the jump operation we have that

$$
\mathbf{f}^{\prime}=d_{\omega}\left(\left\{P^{n+1}(\mathcal{F})\right\}_{n<\omega}\right), \quad \mathbf{g}^{\prime}=d_{\omega}\left(\left\{P^{n+1}(\mathcal{G})\right\}_{n<\omega}\right)
$$

Now suppose that $\mathbf{x} \leq_{\omega} \mathbf{f}^{\prime}, \mathbf{g}^{\prime}$ and that $\mathbf{x}$ is the $\omega$-enumeration degree generated by the sequence $\left(X^{1}, X^{2}, \ldots, X^{k}, \ldots\right)$. Since

$$
\begin{gathered}
\left(X^{1}, X^{2}, \ldots, X^{k}, \ldots\right) \leq_{u}\left\{P^{n+1}(\mathcal{F})\right\}_{n<\omega} \\
\left(X^{1}, X^{2}, \ldots, X^{k}, \ldots\right) \leq_{u}\left\{P^{n+1}(\mathcal{G})\right\}_{n<\omega}
\end{gathered}
$$

we obtain that the sequence $\mathcal{X}=\left(\emptyset, X^{1}, X^{2}, \ldots, X^{k}, \ldots\right)$ is uniformly reducible to the sequences $\mathcal{F}$ and $\mathcal{G}$. Therefore $\mathcal{X} \leq \mathcal{A}_{n}$, for some $n$, i.e.,

$$
\left(\emptyset, X^{1}, X^{2}, \ldots, X^{k}, \ldots\right) \leq_{u}\left(P^{0}\left(\mathcal{A}_{n}\right), P^{1}\left(\mathcal{A}_{n}\right), P^{2}\left(\mathcal{A}_{n}\right), \ldots, P^{k}\left(\mathcal{A}_{n}\right), \ldots\right) .
$$

Therefore

$$
\left(X^{1}, X^{2}, \ldots, X^{k}, \ldots\right) \leq_{u}\left(P^{1}\left(\mathcal{A}_{n}\right), P^{2}\left(\mathcal{A}_{n}\right), \ldots, P^{k}\left(\mathcal{A}_{n}\right), \ldots\right),
$$

which in the terms of the $\omega$-enumeration degrees means that $\mathbf{x} \leq_{\omega} \mathbf{a}_{\mathrm{n}}$.

\section{References}

1. J.Case, Enumeration reducibility and partial degrees, Ann.Math.Log. 2 (1971), 419-439

2. I.N.Soskov, The $\omega$-enumereation degrees, to appear

3. I.N.Soskov, B.Kovachev, Uniform regular enumerations, Math.Struct. in Comp.Sci. 16 (2006), 901-924

4. C.Spector, On degrees of recursive unsolvability, Ann. of Math.(2) 64 (1956), 581592 